\title{
Numerical simulations of heat and mass transfer in the MOVPE process for obtaining high-quality nitride-based semiconductors
}

\author{
J. Skibinski ${ }^{1}$, P. Caban ${ }^{2}$, A. K. Lewandowska ${ }^{3}$, \\ T. Wejrzanowski ${ }^{1}$ \& K. J. Kurzydlowski ${ }^{1}$ \\ ${ }^{1}$ Faculty of Materials Science Engineering, \\ Warsaw University of Technology, Poland \\ ${ }^{2}$ Institute of Electronic Materials Technology, Poland \\ ${ }^{3}$ Faculty of Power and Aeronautical Engineering, \\ Warsaw University of Technology, Poland
}

\begin{abstract}
In the present study heat and mass transfer in a Metal Organic Vapor Phase Epitaxy reactor AIX-200/4RF-S is addressed to better understand the epitaxial growth of gallium nitride (GaN). Existing knowledge about phenomena occurring in the Metal Organic Vapor Phase Epitaxial (MOVPE) process allows the production of high-quality nitride-based semiconductors. However, MOVPE process parameters can vary in certain ranges. The main goal of this study is the optimization of the process and improvement of the quality of crystal obtained. In order to investigate this subject a series of computer simulations have been performed. Numerical simulations of heat and mass transfer in the GaN epitaxial growth process have been performed to determine temperature distribution and velocity profile over the main growth area for various heating temperatures of reagents. Main heat transfer mechanisms during MOVPE process are convection and radiation. Reactor walls are made of quartz to obtain best temperature distribution over the growth area. The study exhibited that heat and mass distribution over the crystal growth area depends on heating temperature and mass flow rate of the inlet gases. Correlation of modeling results with the experiment will allow us to determine an optimal process temperature for obtaining crystals of the highest quality.
\end{abstract}

Keywords: Finite Volume Method, epitaxial growth, gallium nitride. 


\section{Introduction}

Gallium nitride semiconductors have a wide range of applications. They are used for manufacturing numerous semiconductor devices such as High Electron Mobility Transistors (HEMT), Insulated Gate Bipolar Transistor (IGBT). MetalOxide Semiconductor Field-Effect Transistor (MOSFET), Metal Semiconductor Field Effect Transistor (MESFET), Junction gate Field Effect Transistor (JFET), high power blue diodes, LEDs and laser diodes $[1,2]$.

Epitaxy from gaseous phase with use of organometallic compounds is a widely used method for manufacturing gallium nitride. Epitaxial layers obtained in Metal Organic Vapor Phase Epitaxial (MOVPE) growth process create monocrystalline material without grain boundaries [3]. The MOVPE process conditions can vary in certain ranges. Depending on technological parameters different values of surface roughness, dislocation density, resistance and crystal quality can be obtained. Heating temperature, operating pressure and mass flow rate of inlet gases can be optimized to obtain product of best quality. Experimental analysis of the process conditions is very limited mainly due to high temperature and flow disturbance caused by introducing measuring probes [4], therefore modeling is the only solution to understand the process precisely. Numerical model along with experimental data allows to determine correlations between the operating parameters and the quality of the material [5].

This study concerns the influence of heating temperature on the process of crystal growth in MOVPE reactor. In order to investigate this subject a series of computer simulations have been performed. The software used for calculations was ANSYS Fluent.

\section{Case study}

To study the influence of process heating temperature on crystal growth, a model of the MOVPE reactor AIX-200/4RF-S has been created. The MOVPE reactor and its model are shown in Figures 1 and 2, respectively.

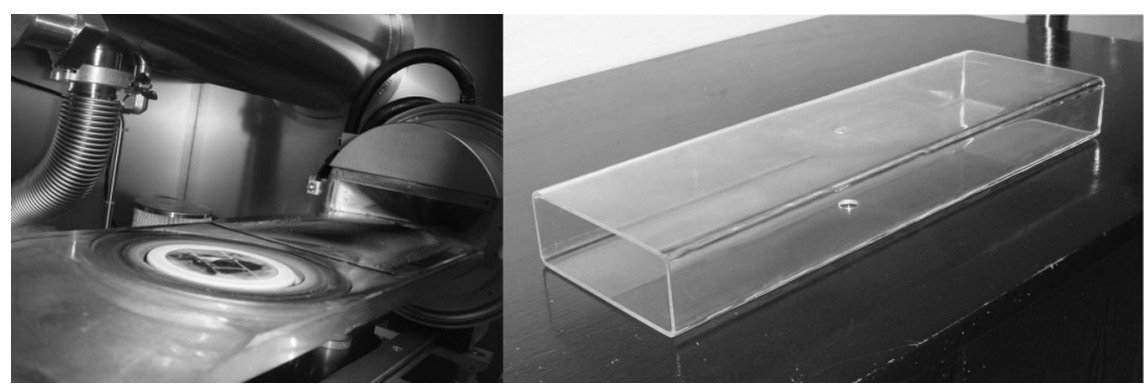

Figure 1: Chamber of MOVPE reactor. 


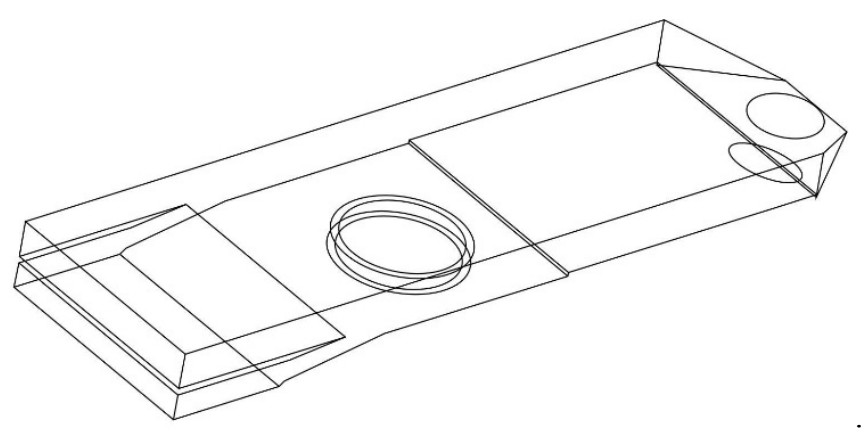

Figure 2: Schematic model of MOVPE reactor.

The disc on which silicon carbide base is placed is made of graphite, and is induction heated during the process. Experimental measurements show that the graphite disc has constant temperature during the crystal growth process. Walls of the reactor are made of quartz. This material was chosen due to its best properties regarding radiative heat transfer mechanism. The inlet gases are introduced to the reactor in room temperature with two separate inlets. Upper inlet supplies hydrogen $\left(\mathrm{H}_{2}\right)$ and trimethylgallium $\left(\left(\mathrm{CH}_{3}\right)_{3} \mathrm{Ga}\right)$, while mixture of hydrogen $\left(\mathrm{H}_{2}\right)$ and ammonia $\left(\mathrm{NH}_{3}\right)$ are introduced to the reactor through lower inlet. Mixing of the gases takes place just before the crystal growth area. Additionally, the heated disc is rotating during the process, which allows to obtain more homogeneous growth rate on the silicon carbide plate and assures more homogenous distribution of the reagents over the chemical reactions area. The reactor chamber is placed in a pipe, where hydrogen at room temperature is constantly injected to cool the outer walls of the reactor.

A finite volume mesh of 300,000 triangular elements has been created for the geometry of the reactor (Figure 3 ). The mesh is refined over the chemical reactions area, since it's the area of biggest importance, and mesh should be the most accurate above it.

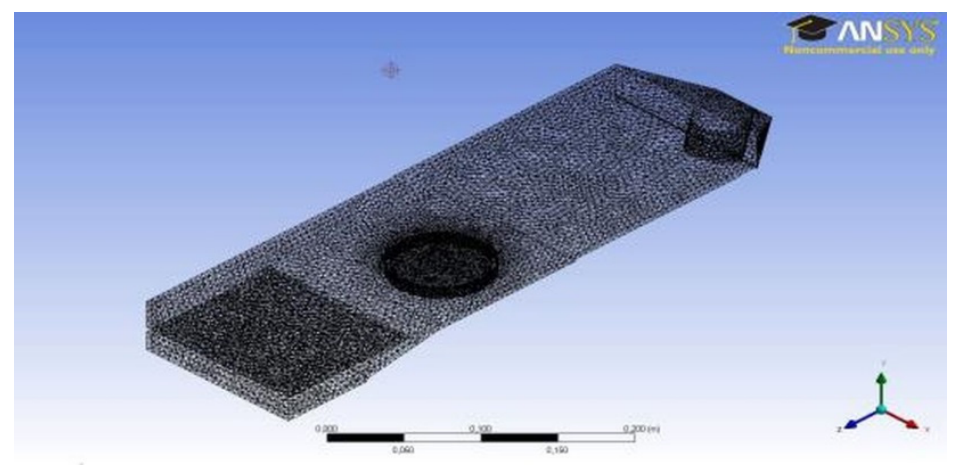

Figure 3: Finite element mesh of the epitaxy reactor. 
ANSYS Fluent software, used for calculations, solves a set of differential equations describing mass and heat transfer. The governing equations in Computational Fluid Dynamics problems are mass, momentum and energy equations (1).

$$
\frac{\partial}{\partial t}(\rho E)+\nabla \cdot(\underset{v}{\rightarrow}(\rho E+p))=\nabla \cdot\left(k_{e f f} \nabla T-\sum_{j} h_{j} \underset{J_{j}}{\rightarrow}+\left(\overline{\bar{\tau}}_{e f f} \cdot \underset{v}{\rightarrow}\right)+S_{h}\right.
$$

In case of MOVPE process modeling radiative heat transfer has to be taken into account. The equation of radiant heat flux is given by equation (2).

$$
Q_{\text {rad }}=\sigma\left(T_{\max }^{4}-T_{\min }^{4}\right)
$$

The mixture of gases in the numerical model is simulated as an ideal gas. The density for such case is described by the Clapeyron formula (3).

$$
\rho=\frac{\mathrm{pM}}{\mathrm{RT}}
$$

Main heat transfer mechanisms for the epitaxial growth process are convection and radiation. As a conclusion of the experimental measurements, induction heating is replaced in the computer model by assumption of constant temperature of the graphite disc.

\section{Results}

To compare the influence of specific parameters on the distribution of heat and mass inside the reactor, a series of simulations was performed. Parameters studied in this work were heating temperature, hydrogen mass flow rate and rotational speed of the disc. Only one of the parameters was changed in each simulation series.

The investigated heating temperature range was 1073 to $1473 \mathrm{~K}$ and the inlet gases temperature was $300 \mathrm{~K}$. Process parameters for the chosen temperature range are listed in Table 1.

Table 1: Process parameters for studying temperature influence.

\begin{tabular}{cccccc}
\hline Pressure & Temperature & $\begin{array}{c}\text { TMGa } \\
\text { mass flow } \\
\text { rate }\end{array}$ & $\begin{array}{c}\text { Hydrogen } \\
\text { mass flow } \\
\text { rate }\end{array}$ & $\begin{array}{c}\text { Ammonia } \\
\text { mass flow } \\
\text { rate }\end{array}$ & $\begin{array}{c}\text { Rotation } \\
\text { speed of } \\
\text { the disc }\end{array}$ \\
\hline $50 \mathrm{mbar}$ & $1073 \mathrm{~K}$ & $20 \mathrm{sccm}$ & $6000 \mathrm{sccm}$ & $2000 \mathrm{sccm}$ & $4 \mathrm{rad} / \mathrm{s}$ \\
$50 \mathrm{mbar}$ & $1273 \mathrm{~K}$ & $20 \mathrm{sccm}$ & $6000 \mathrm{sccm}$ & $2000 \mathrm{sccm}$ & $4 \mathrm{rad} / \mathrm{s}$ \\
$50 \mathrm{mbar}$ & $1383 \mathrm{~K}$ & $20 \mathrm{sccm}$ & $6000 \mathrm{sccm}$ & $2000 \mathrm{sccm}$ & $4 \mathrm{rad} / \mathrm{s}$ \\
$50 \mathrm{mbar}$ & $1473 \mathrm{~K}$ & $20 \mathrm{sccm}$ & $6000 \mathrm{sccm}$ & $2000 \mathrm{sccm}$ & $4 \mathrm{rad} / \mathrm{s}$ \\
\hline
\end{tabular}

Calculated temperature distribution inside the MOVPE reactor and over the epitaxial growth area are shown in Figures 4 and 5, respectively. 


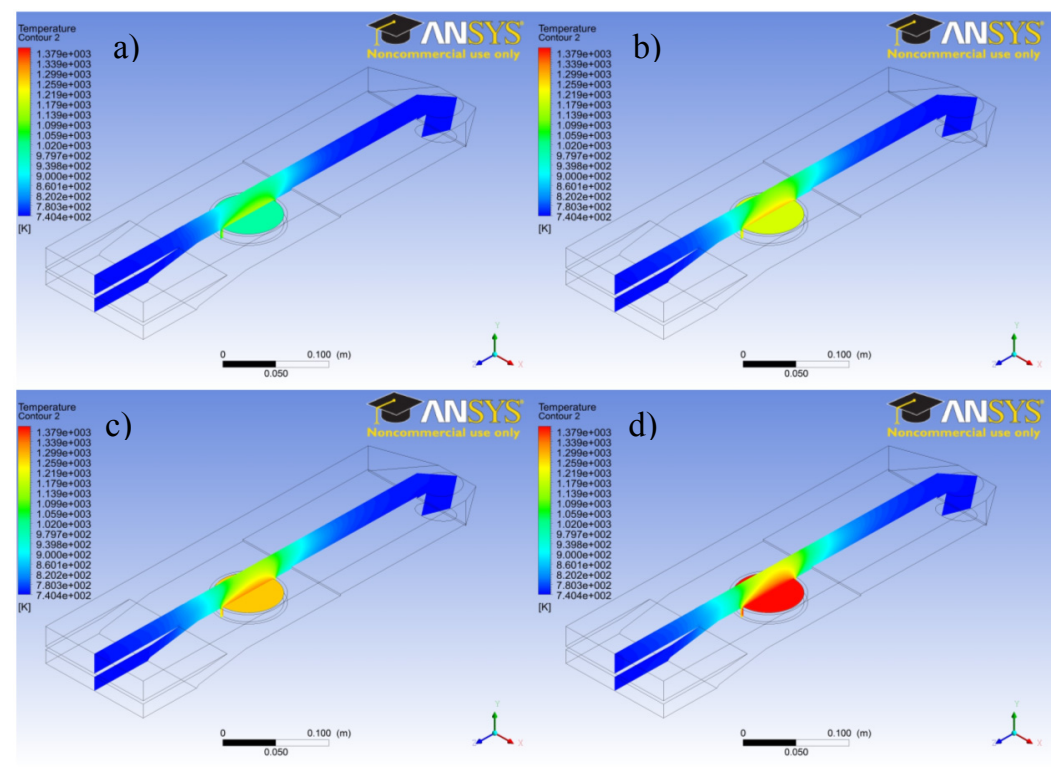

Figure 4: Temperature distribution in MOPVE reactor for temperatures: a) $1073 \mathrm{~K}$; b) $1273 \mathrm{~K}$; c) $1383 \mathrm{~K}$; d) $1473 \mathrm{~K}$.

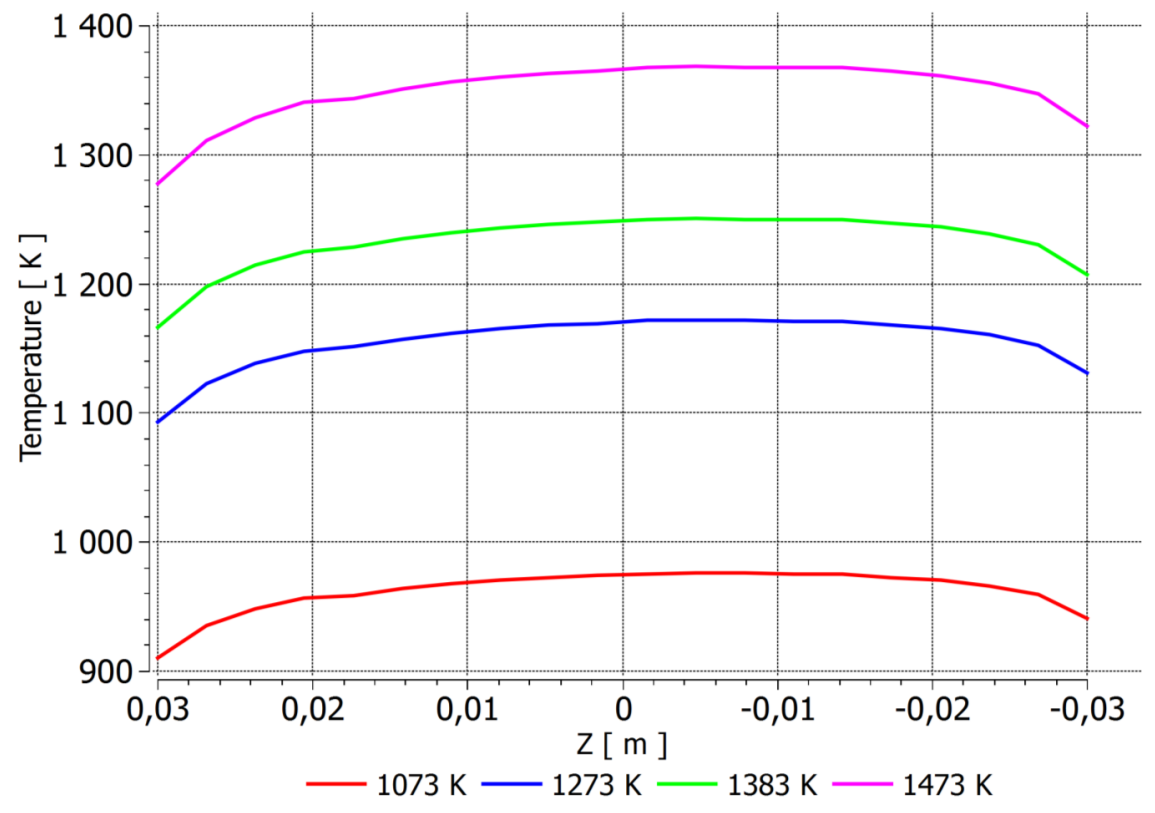

Figure 5: Temperature distribution over the epitaxial growth area of MOVPE reactor for processes in temperatures $1073,1273,1383$ and $1473 \mathrm{~K}$. 
The next set of simulations was performed in order to investigate the influence of hydrogen mass flow rate on the epitaxial process. Process parameters for chosen hydrogen mass flow rates are listed in Table 2.

Table 2: Process parameters for studying hydrogen mass flow rate influence.

\begin{tabular}{cccccc}
\hline Pressure & Temperature & $\begin{array}{c}\text { TMGa } \\
\text { mass flow } \\
\text { rate }\end{array}$ & $\begin{array}{c}\text { Hydrogen } \\
\text { mass flow } \\
\text { rate }\end{array}$ & $\begin{array}{c}\text { Ammonia } \\
\text { mass flow } \\
\text { rate }\end{array}$ & $\begin{array}{c}\text { Rotation } \\
\text { speed of } \\
\text { the disc }\end{array}$ \\
\hline $50 \mathrm{mbar}$ & $1383 \mathrm{~K}$ & $20 \mathrm{sccm}$ & $1500 \mathrm{sccm}$ & $2000 \mathrm{sccm}$ & $5 \mathrm{rad} / \mathrm{s}$ \\
$50 \mathrm{mbar}$ & $1383 \mathrm{~K}$ & $20 \mathrm{sccm}$ & $3000 \mathrm{sccm}$ & $2000 \mathrm{sccm}$ & $5 \mathrm{rad} / \mathrm{s}$ \\
$50 \mathrm{mbar}$ & $1383 \mathrm{~K}$ & $20 \mathrm{sccm}$ & $6000 \mathrm{sccm}$ & $2000 \mathrm{sccm}$ & $5 \mathrm{rad} / \mathrm{s}$ \\
$50 \mathrm{mbar}$ & $1383 \mathrm{~K}$ & $20 \mathrm{sccm}$ & $1200 \mathrm{sccm}$ & $2000 \mathrm{sccm}$ & $5 \mathrm{rad} / \mathrm{s}$ \\
\hline
\end{tabular}

Velocity profile of gases inside the MOVPE reactor for different $\mathrm{H}_{2}$ mass flow rates is shown in Figure 6.

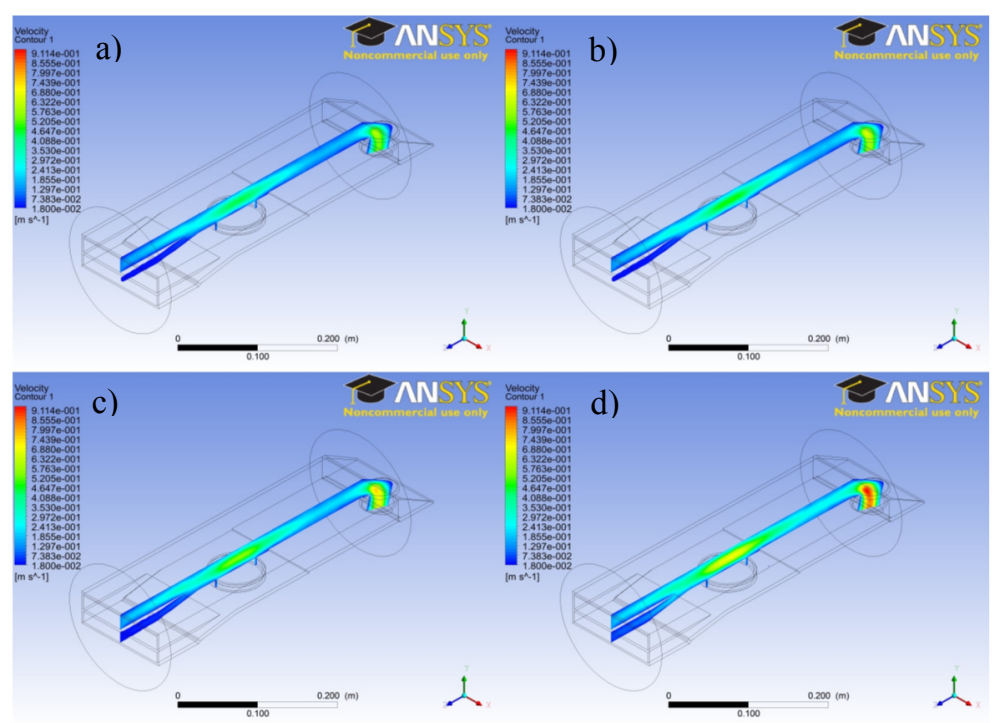

Figure 6: Velocity distribution in epitaxy reactor MOVPE for the hydrogen mass flows: a) $1500 \mathrm{sccm}$; b) $3000 \mathrm{sccm}$; c) $6000 \mathrm{sccm}$; d) $12000 \mathrm{sccm}$.

The temperature distribution over the crystal growth area changes depending on hydrogen mass flow rate (Figure 7). 


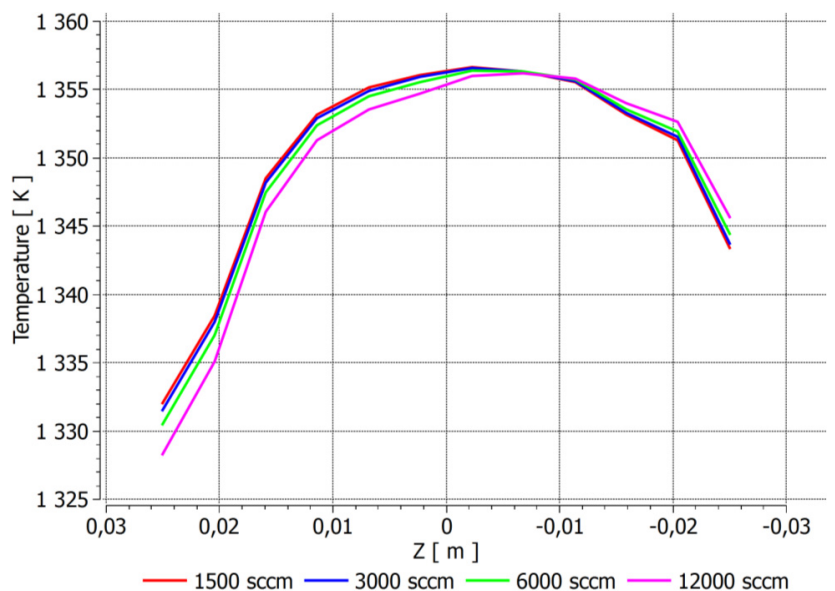

Figure 7: Temperature distribution over the epitaxial growth area for processes with $\mathrm{H}_{2}$ mass flow rates of $1500,3000,6000$ and $12000 \mathrm{sccm}$.

The caluclations have shown that the $\mathrm{H}_{2}$ mass flow rate significantly influences the distribution of the reagents over the crystal growth area (Figure 8). Hower the temperature distribution inside the reactor is not influenced by the value of rotational speed.

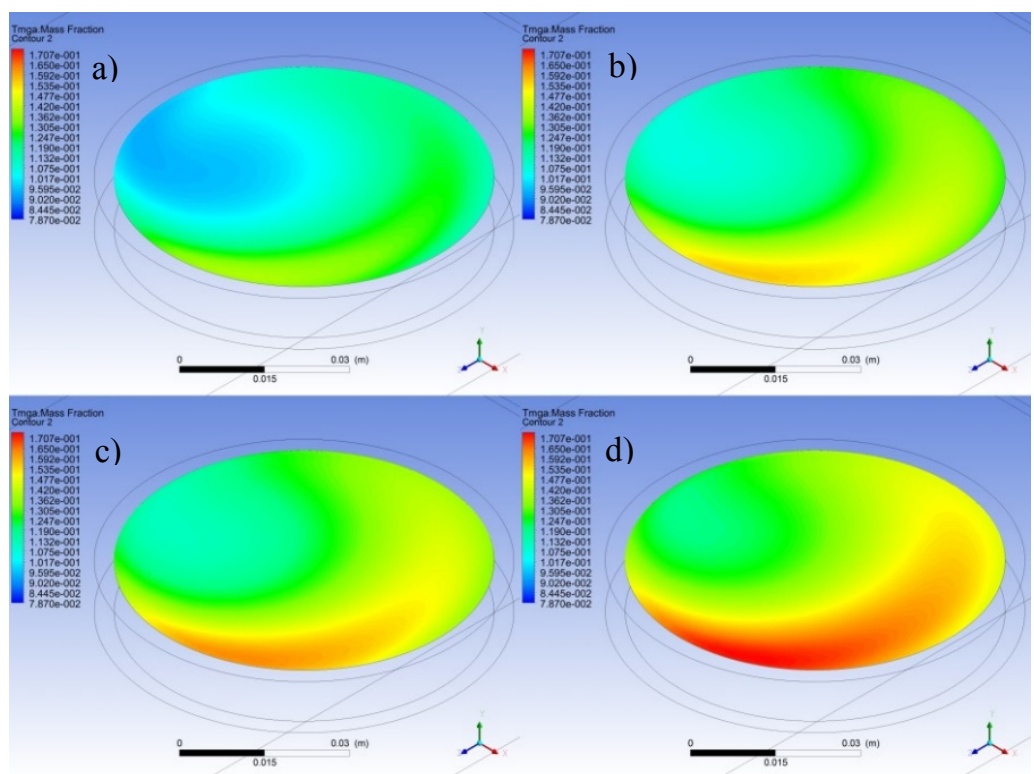

Figure 8: Trimethylgallium mass fraction distribution over the gallium nitride (GaN) crystal growth area: a) $1500 \mathrm{sccm}$; b) $3000 \mathrm{sccm}$; c) $6000 \mathrm{sccm}$; d) $12000 \mathrm{sccm}$. 
The influence of rotational speed of the disc on heat and mass transfer distribution inside the reactor was also studied.

\section{Conclusions}

Heat and mass transfer distribution in gallium nitride MOVPE process was studied. Results exhibited, that regardless the temperature of the heating element, temperature and density of reagents over the crystal growth area are not homogeneous. The most important conclusion from heat and mass transfer calculations is that the homogeneity of reagents distribution over the crystal growth area changes depending on hydrogen mass flow rate. Including chemical reactions in numerical model will allow to directly determine the surface deposition rate of gallium nitride. Including chemical reactions in numerical model together with experimental verification will allow to determine the dependence between the change of the temperature gradient over the crystal growth area and the quality of obtained crystal. Determining a valid range of temperature and mass fraction of gases during epitaxial crystal growth will allow to optimize process parameters and to obtain crystals of best quality.

\section{Acknowledgement}

This work was supported by the European Regional Development Fund within the Innovative Operational Programme in the frame of project SICMAT (Grant No. POIG.01.03.01.14-155/09).

\section{References}

[1] Park, Yoon Soo. "SiC materials and devices", Semiconductors and Semimetals, Vol. 52, San Diego: Academic press.

[2] N.G. Wright, A.B. Horsfall, K. Vasiilevski. "Prospects for SiC electronics and sensors" Materials today vol. 11 (2008) Nr 1-2.

[3] P.A. Caban et al. "Effect of growth pressure on coalescence thickness and crystal quality of GaN deposited on 4H-SiC", Journal of Crystal Growth 315 (2011) 168-173.

[4] W. Strupinski et al. "Graphene Epitaxy by Chemical Vapor Deposition on SiC" Nano Letters 11 (2011) 1786-1791.

[5] S.A. Safvi, J.M. Redwing, M.A. Tischler, T.F. Kuech, "GaN Growth by Metallorganic Vapor Phase Epitaxy: Comparison of Modeling and Experimental Measurements" Journal of Electrochemical Society Vol. 144 (1997) 5, 1789-1796.

[6] F.A. Ponce et al. "Microstructure of GaN epitaxy on SiC using Aln buffer layers" Applied Physics Letters 67 (1995) 410. 